\title{
Radical Polymerization of Furan with Maleic Anhydride through the Diels-Alder Adduct
}

\author{
Bunzo Kamo, Isamu Morita, Satoshi Horie, \\ and Shijo Furusawa \\ Department of Industrial Chemistry, Faculty of Science and Engineering, \\ Chuo University, Bunkyo-ku, Tokyo, Japan.
}

(Received August 30, 1973)

\begin{abstract}
Equimolar reactions of furan (F), 2-methylfuran (MF), and 2,5-dimethylfuran (DMF) with maleic anhydride in acetone- $d_{6}$ at $60^{\circ} \mathrm{C}$ were directly followed by NMR spectra. The rates of formation of the two isomers, endo and exo adducts, formed by the Diels-Alder reactions were in the order: endo form; DMF $>\mathrm{F}>\mathrm{MF}$; exo form; DMF $>$ MF $>$ F. The maximum conversions for the endo isomers observed in all cases suggest the isomerization of endo adducts to exo isomers. The polymers formed were not $1: 1$ alternating copolymers, but homopolymers resulting from the Diels-Alder adducts of exo form. The complex formed in the reaction system might be the active intermediate for the Diels-Alder reaction, but not for the polymerization reaction.

The reaction of furfuryl methacrylate with maleic anhydride provides another example where the Diels-Alder reaction participates in the polymerization reaction.

KEY WORDS Radical Polymerization / Furan Derivatives / DielsAlder Reaction / endo Adduct / exo Adduct / Maleic Anhydride / Polymerization of Adduct / Charge-Transfer Complex / Furfuryl Methacrylate / NMR Spectra /
\end{abstract}

Two of the authors previously reported that furan (F), 2-methylfuran (MF), and 2,5-dimethylfuran (DMF) ordinarily took part in the DielsAlder reaction with maleic anhydride (MAH) in the presence of a radical initiator, followed by the polymerization of the resulting adducts. ${ }^{1}$

Recently, Butler, et al., ${ }^{2}$ reported that a $1: 1$ alternating copolymer between $\mathrm{F}$ and $\mathrm{MAH}$ was readily obtained by a free radical initiator in benzene solutions of the comonomers.

However, characterizations of the resulting copolymers by means of NMR and IR spectra analyses were not performed.

The formation of a $1: 1$ alternating copolymer was suggested from the following two data: 1) the copolymer composition evaluated from the high-frequency titration values and elementary analysis of the polymer; 2) the presence of the charge-transfer complex from $\mathrm{F}$ and $\mathrm{MAH}$. These results can also be well explained by our suggestion that the polymerization product was not a copolymer, but the polymer resulting from the Diels-Alder adducts. The present paper will compare our experimental results with those of Butler, et al., ${ }^{2}$ and will clarify our position that furan derivatives and MAH do not undergo free radical copolymerization to yield a $1: 1$ alternating copolymer, but ordinarily take part in the Diels-Alder reaction, after which the resulting adducts are homopolymerized by radical initiators.

\section{EXPERIMENTAL}

\section{Materials}

Commercially available F, MF, and DMF which were prepared from the Wolff reduction of furfural and 5-methylfurufural, respectively, were purified by distillation, dried over metallic sodium, and redistilled under a nitrogen atmosphere.

MAH was a commercial reagent and was purified by reduced distillation. F-MAH, MF-MAH, and DMF-MAH adducts were prepared in ether at $35^{\circ} \mathrm{C}$ from the corresponding furans and $\mathrm{MAH}$, and recrystallized from 
ethyl acetate. Exo-7-oxabicyclo-(2,2,1)-hexane5,6-dicarboxylic anhydride (HPA) was prepared by catalytic hydrogenation of exo-7-oxabicyclo(2,2,1)-2-hexane-5,6-dicarboxylic anhydride (TPA) and purified by recrystallization from ligroin; mp $116^{\circ} \mathrm{C}$ (lit..$\left.^{3} 116^{\circ} \mathrm{C}\right)$.

Polymerization Procedures and Purification of
Polymer Polymer

The required amounts of MAH and initiator were placed in an ampoule which had previously been filled with dry nitrogen. The furans and solvent were introduced into the ampoule, which was then connected to a vacuum line, degassed at liquid nitrogen temperature, and sealed off. This ampoule was finally set in a water bath controlled at a constant temperature.

After a given period of time the contents of the ampoule were poured into a large amount of ethyl ether to precipitate the resulting polymer. The precipitated polymer was extracted with ether by Soxhlet extractor for $24 \mathrm{hr}$.

The residue was washed thoroughly with ethyl acetate and dried at $60^{\circ} \mathrm{C}$ in vacuo.

\section{Analysis of Polymer Composition}

In order to determine the anhydride content, the polymer was titrated with sodium methoxide in nonaqueous solvent (acetone), according to the method of Fritz, et $a l^{4}$ The polymer composition was calculated on the basis of the titration values and the $\mathrm{C}-\mathrm{H}$ elementary analysis.

Molecular Weight. The molecular weight of the polymer was measured by a vapor pressure osmometer (Hitachi, model 115) in acetone at $35^{\circ} \mathrm{C}$.

NMR and IR Spectra Measurements. NMR spectra were taken on a JEOL-PS-100 MHz instrument in acetone- $d_{6}$, using tetramethylsilane as an internal standard.

IR spectra were recorded with a Hitachi Perkin-Elmer Model 225 on a $\mathrm{KBr}$ pellet.

$D T A$ analysis. Differential thermal analysis of the polymer was carried out on a Rigaku Denki Model K 10206.

\section{RESULTS AND DISCUSSION}

The results of the radical polymerizations of the furan derivatives with MAH are summarized in Table I.

Throughout the reaction, the polymerization proceeded heterogeneously in benzene solution and homogeneously in acetone. The conversions increased in the following order: $\mathrm{F}<\mathrm{MF}<$ DMF. The exo isomers of the Diels-Alder adducts resulting from the furan derivatives and MAH were also successfully polymerized to give polymers similar to those from the corresponding addents. The yields of the "adduct polymers,"

Table I. Results of polymerization

\begin{tabular}{lccccccc}
\hline Monomer & $\begin{array}{c}\text { Molar } \\
\text { ratio }\end{array}$ & Solvent & $\begin{array}{c}\text { Time, } \\
\text { hr }\end{array}$ & $\begin{array}{c}\text { Yield, } \\
\text { wt\% }\end{array}$ & $\begin{array}{c}C_{\%}^{\text {a }} \\
\%\end{array}$ & $\begin{array}{c}\text { MAH mol } \\
\text { fract, \% }\end{array}$ & $\begin{array}{c}\text { Molecular } \\
\text { weight }^{\mathrm{b}}\end{array}$ \\
\hline \multirow{3}{*}{ F-MAH } & $1: 1$ & Benzene & 20 & 26 & 56.43 & 57.1 & 8300 \\
& $1: 1$ & Acetone & 20 & 24 & 56.53 & 55.5 & 5800 \\
& $3: 7$ & Acetone & 4 & 11 & 55.37 & 62.1 & 4300 \\
& $7: 3$ & Acetone & 8 & 8 & 56.43 & 56.1 & 1240 \\
F-MAH Adduct & $6: 4$ & Acetone & 4 & 5 & 56.52 & 56.9 & 3800 \\
\hline \multirow{2}{*}{ MF-MAH } & & Benzene & 20 & 29 & 56.93 & 50.1 & 4400 \\
\hline DMF-MAH & $1: 1$ & Acetone & 20 & 32 & 56.97 & 54.2 & 2700 \\
\hline
\end{tabular}

a Determined by elementary analysis.

b Determined by VPO in acetone at $30^{\circ} \mathrm{C}$.

c No catalyst.

Cat: AIBN, $2 \mathrm{~mol} \%$; temp, $70^{\circ} \mathrm{C}$; total monomer, $4.5 \times 10^{-2} \mathrm{~mol}$. 
obtained by the radical polymerizations of the exo isomers of the Diels-Alder adducts, are slightly better than those of the "addent polymers" from the addents of the Diels-Alder reactions of the furan derivatives with MAH.

Both the addent and adduct polymers were white powders which were soluble in acetone and aqueous alkaline solution and had a maximum molecular weight in the region of $10 .{ }^{4}$

They were thermally stable, as shown in Figure 1. No thermal changes were observed at $52^{\circ} \mathrm{C}$, which is the melting point of $\mathrm{MAH}$, and at $\mathrm{A}$, which is the decomposition point of the exo isomer of the Diels-Alder adduct resulting from $\mathrm{F}$ and $\mathrm{MAH}$. The first exothermic loss in weight of the polymer appeared at the elevated temperature of $245^{\circ} \mathrm{C}$.

The NMR and IR spectra of the polymer obtained by the radical polymerization of $F$ with MAH are shown in Figures 2 and 11,

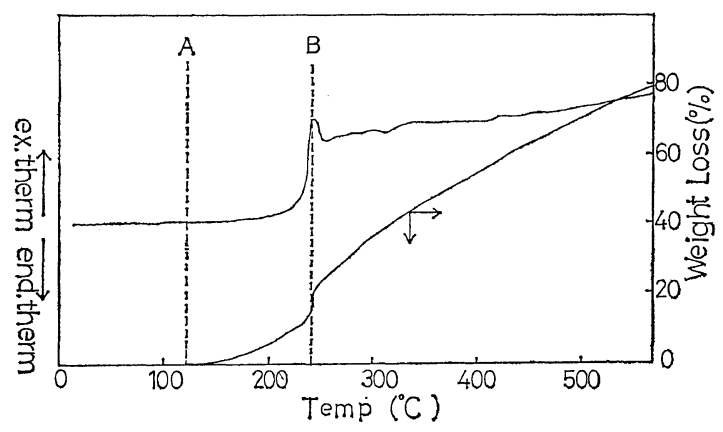

Figure 1. Thermograms of the adduct polymer resulting from $\mathrm{F}$ with $\mathrm{MAH}$.

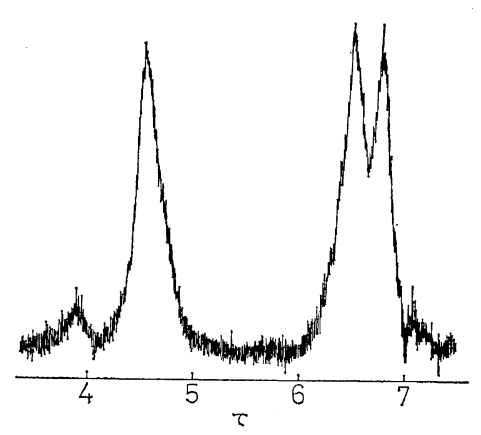

Figure 2. NMR spectrum of the polymer obtained by the radical polymerization of $\mathrm{F}$ with $\mathrm{MAH}$, in acetone- $d_{6}(5 \mathrm{wt} / \mathrm{vol} \%)$ at $50^{\circ} \mathrm{C}(100 \mathrm{MHz})$, using TMS as an internal standard. respectively, while the chemical shifts of the polymer are summarized in Table II.

The NMR and IR spectra of the adduct polymer resulting from $\mathrm{F}$ and $\mathrm{MAH}$ are exactly identical with those of the addent polymer.

The similarity of the spectra between the adduct and addent polymer was also observed in the MF-MAH system as well as in the DMF-MAH one.

From the identity of their spectra, it is clear that the adduct polymer and the addent polymer contain essentially the same structural units. It seems reasonable that the polymers have repeating units of the type (I).

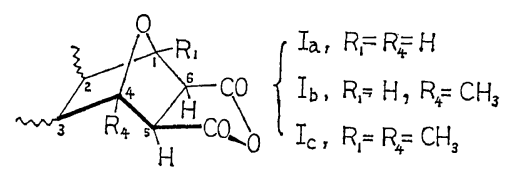

(I)

Evidence for the presence of 2,3-disubstituted HPA (exo form) repeat units in these polymers is offered by the NMR and IR spectra.

The chemical shifts of TPA, exo and endo isomers of the Diels-Alder adducts resulting from the furan derivatives and $\mathrm{MAH}$, synthesized as model compounds of the polymer formed, are summarized in Table II.

As shown in Figure 2, the NMR spectrum of the addent polymer resulting from $F$ and MAH shows three main peaks, at $4.59,6.51$, and $6.80 \tau$. The peaks at $4.59 \tau$ may be assigned

Table II. Chemical shifts $(\tau)$ of the polymer and its model compounds in acetone- $d_{6}$ at $22^{\circ} \mathrm{C}$

\begin{tabular}{llllll}
\hline $\begin{array}{c}\text { Reaction } \\
\text { system }\end{array}$ & \multicolumn{1}{c}{ Sample } & $\begin{array}{l}\mathrm{H}_{2}, \\
\mathrm{H}_{3}\end{array}$ & $\begin{array}{l}\mathrm{H}_{1}, \\
\mathrm{H}_{4}\end{array}$ & $\begin{array}{l}\mathrm{H}_{5}, \\
\mathrm{H}_{6}\end{array}$ & $\mathrm{CH}_{3}$ \\
\hline \multirow{4}{*}{ F-MAH } & Polymer & 6.80 & 4.59 & 6.51 & - \\
& exo Adduct & 3.37 & 4.65 & 6.68 & - \\
& endo Adduct & 3.28 & 4.63 & 6.08 & - \\
& HPA & 8.22 & 5.05 & 6.54 & - \\
\hline \multirow{4}{*}{ MF-MAH } & Polymer & 6.71 & 4.89 & 6.60 & 8.34 \\
& exo Adduct & 3.63 & 4.12 & 5.98 & 7.78 \\
& endo Adduct & 3.60 & 4.04 & 5.29 & 7.78 \\
\hline \multirow{4}{*}{ DMF-MAH } & Polymer & 6.65 & - & 6.89 & 8.58 \\
& exo Adduct & 3.64 & - & 6.68 & 7.82 \\
& endo Adduct & 3.63 & - & 6.36 & 7.82 \\
\hline
\end{tabular}


to the methine protons at the 1- and 4-positions in the structure of the type (Ia), from its correspondence to that in the spectra of the model compounds (Table II). From our early study ${ }^{11}$ of the alternating copolymerization of 2,5dihydrofuran derivatives with MAH, two methine protons of 2,5-dihydrofuran units in the copolymer were found to appear at ca. $7 \tau$. Therefore, the peak at $6.80 \tau$ may be assigned to the methine protons in the main chain of the polymer consisting of the repeat units (Ia).

From Table II, it is clear that in all the exo isomers of the model compounds the methine protons at the 5- and 6-positions show peaks at $6.6-6.8 \tau$, whereas those of the endo isomers resonate at ca. $6 \tau$. Therefore, the signal of the exo methine protons of the resulting polymer may be expected to appear at a higher magnetic field than that of the endo methine protons.

Consequently, in the spectrum of Figure 2, the peaks at $6.80 \tau$ should be assigned to the methine protons at the 5- and 6-positions in the polymer with exo configuration (Ia).

In addition to these spectroscopic assignments, the elementary analyses of the resulting polymer support the assertion that it has recurring units of the type (Ia). The polymer composition based on Carbon-Hydrogen analysis was found to be equimolar over a wide range of monomer feed ratio. The nitrogen content was also employed for the composition analysis of the product obtained by refluxing the polymer with aniline under $\mathrm{N}_{2}$ atmosphere. The polymer composition was calculated on the basis of the $\mathrm{N}$ content for $(\mathbf{X})$, which represents the suspected repeating units.

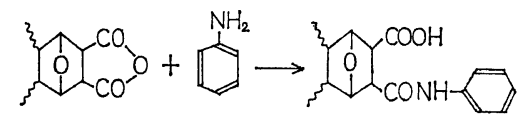

(X)

Anal. Calcd for $\mathrm{C}_{14} \mathrm{H}_{13} \mathrm{NO}_{4}:$ N, 5.41. Found: N, 5.38\%.

The MAH content in the polymer was successfully estimated by titrating the polymer with sodium methoxide in nonaqueous solvent (i.e., acetone), using thymol blue as an indicator, according to the method of Fritz, et al. ${ }^{4}$ The titration value suggests that the polymers are
98\% composed of repeating units.

From the above experimental results, it may be concluded that the polymer consists of equimolar amounts of $\mathrm{F}$ and $\mathrm{MAH}$, and has repeating units of the type (Ia).

On the other hand, Butler, et al., ${ }^{2}$ have previously suggested that $F$ and $\mathrm{MAH}$ undergo free radical copolymerization to yield a product of approximately $1: 1$ comonomer composition (II).

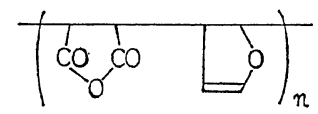

(II)

The following three experimental results were shown as evidence for the formation of the alternating copolymer: ${ }^{2)}$ (1), $1: 1$ comonomer composition calculated from the $\mathrm{C}-\mathrm{H}$ elementary analysis of the polymerization products; (2), $1: 1$ comonomer composition determined by the anhydride content calculated from the highfrequency titration values; (3), the formation of 1: 1 charge-transfer complex from $F$ and $M A H$, suspected to account for the $1: 1$ alternating sequence of the monomers in the product.

The former two results, however, can be explained by our suggestion that the product has repeat units of the type (Ia).

Therefore, it is impossible from the polymer composition analysis alone to decide whether a true repeating unit of the polymer is (I) or (II). Moreover, the geometry of the charge-transfer complex is unknown and its exact role is not clarified yet. It is obvious that the presence of the charge-transfer complex species with a stoichiometry of $1: 1$ does not necessarily guarantee the formation of the alternating copolymer. To elucidate the role of the complex in the polymerization of $\mathrm{F}$ with $\mathrm{MAH}$, the following spectroscopic measurements were carried out.

\section{Characterization of Charge-Transfer Complex}

When a chloroform solution of $\mathrm{F}$ was added to a solution of MAH, there appeared a charge transfer complex band in the near-ultraviolet region $\left(\mathrm{UV}_{\max } ; 284 \mathrm{~nm}\right)$. The complex stoichiometry of $1: 1$ was determined by the authors ${ }^{1}$ and by Butler, et al., ${ }^{2}$ using the continuous 
variation method of Job. ${ }^{5}$ It was found that MF and DMF had analogously formed chargetransfer complexes with absorption maxima at 305 and $330 \mathrm{~nm}$, respectively, which also had a stoichiometry of $1: 1$ (Figure 3).

UV scans for a mixed solution of furans and MAH were recorded at 10 -min intervals. The absorbances due to the complex species were found to decrease with time, as shown in Figure 4.

The resulting absorbances against time are shown in Figure 5.

As the complexes are probably the transitory contact charge transfer ones, ${ }^{6}$ the concentrations of the complex were promptly attained at equilibrium as soon as the donor and the acceptor were mixed together. In these cases, the absorbance decay may in general not be observed. It is well known that vinyl ethers are polymerized with $\mathrm{MAH}$ in the presence of a radical initiator to give $1: 1$ alternating copolymers, in which the charge-transfer complex of $1: 1 \mathrm{com}-$ position formed in the reaction system participates in the initiation reaction, and the structure of the complex was proposed. ${ }^{7}$ In the 2,3dihydrofuran-MAH system, ${ }^{8}$ spontaneous polymerization proceeded at room temperature; here the absorbance of the charge-transfer complex changed with time.

In the present system, however, the polymerization reaction did not take place without decomposition of the radical initiator, but the Diels-Alder reaction occurred at a fairly high rate. Therefore, the complex formed in the furans and MAH system might be the active

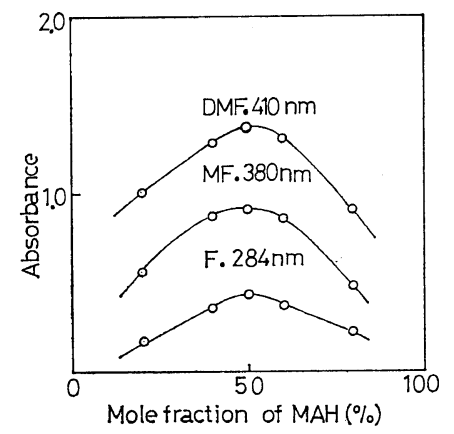

Figure 3. Continuous variation method applied to F-, MF-, and DMF-MAH complexes; [Furan deriv. $]+[\mathrm{MAH}], 1.004 \mathrm{~mol} / l$, room temperature.

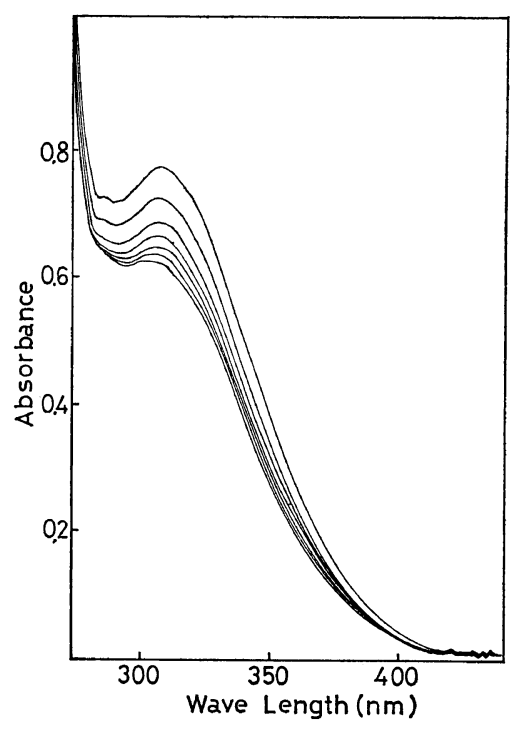

Figure 4. UV scans for a mixed solution of MF and MAH recorded at 10-min intervals: [MF], 0.5 $\mathrm{mol} / l$; [MAH], $0.05 \mathrm{~mol} / l$; measured at $23^{\circ} \mathrm{C}$.

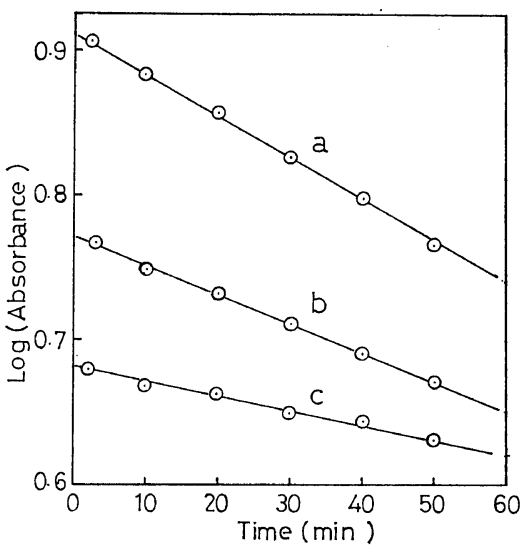

Figure 5. Variations with time of max absorbance due to complexes in chloroform solution: a, DMF-MAH; b, MF-MAH; c, F-MAH; [F], [MF], [DMF], $0.5 \mathrm{~mol} / l ;$ [MAH], $0.05 \mathrm{~mol} / l$.

intermediate for the Diels-Alder reaction, but not for the polymerization reaction; that is, the complex formed in the reaction system does not play any important role in the initiation of the polymerization reaction. Accordingly, another polymerization mechanism for the radical polymerization of furan derivatives with MAH was needed. 


\section{Polymerization Mechanism}

Conceivable reaction processes between furans and MAH in the presence of free radical initiator were summarized schematically in Figure 6.

(1) Diels-Alder reaction: It is well known ${ }^{3}$ that furans and MAH readily take part in the Diels-Alder reaction to yield quantitatively the adduct of $1: 1$ composition. The adducts have two possible configurations, endo and exo forms. ${ }^{9}$ The exo isomers of F, MF, and DMF were unstable crystalline, soluble in acetone, and quantitatively reverted to the addents at 110 , 58 , and $78^{\circ} \mathrm{C}$, respectively. There is a suspicion that they might be loose molecular compounds. However, the fact that the adducts change into extremely stable compounds by reducing a double bond of the furan nuclei suggest the formation of the true adducts. For example, by reducing TPA over platinum oxide to HPA, it came to melt at $116^{\circ} \mathrm{C}$ without decomposition. That is, the strength of the bond between the 1- and 6-positions as well as the 4- and 5-positions increases much more strongly in HPA than in TPA. Moreover, an oxygen bridge of the adducts is stable under temperature changes. The Diels-Alder adduct was isolated in 30$40-\%$ yield as a by-product of the polymerization reaction; it was removed by extraction with ethyl ether by a Sohxlet extractor, followed by drying at $60^{\circ} \mathrm{C}$ in vacuo. IR and NMR spectra of the polymer were significantly different from those of the adduct.

(2) Diels-Alder polymerization: Cyclopentadiene is usually dimerized itself at room tem-

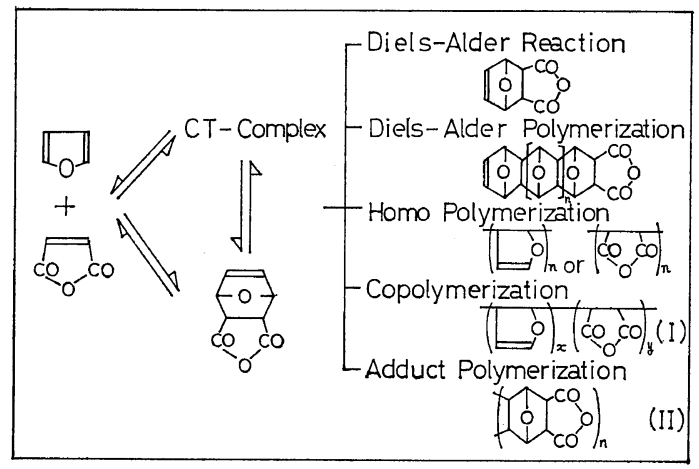

Figure 6. Concievable reaction processes between furan derivatives and $\mathrm{MAH}$ in the presence of free radical initiator. perature, and the resulting double bond of the norbornene type as a dienophile reacts with monomer as a diene to form polymer by the Diels-Alder reaction. ${ }^{10}$

F itself, however, had no possibility of dimerization.

Therefore, the Diels-Alder polymerization could not take place in the present system.

(3) Homopolymerization of $\mathrm{F}$ and/or MAH: Furan derivatives did not homopolymerize by radical initiators and MAH was difficult to polymerize under the present conditions.

(4) Radical copolymerization: We previously confirmed that furan derivatives did not copolymerize with styrene and other vinyl monomers in the presence of various radical initiators. ${ }^{1}$

Moreover, it could not be acceptable for the mechanism of the radical polymerization of furan derivatives with MAH that the chargetransfer complex species of a stoichiometry of 1:1 "homopolymerizes," resulting in the high degree of regularity of composition in the copolymer, as suggested by Butler, et $a .^{2}$

(5) Radical polymerization through the Diels-Alder adduct: Now, we propose a possible polymerization mechanism, "radical polymerization through the Diels-Alder adduct," for the formation of the polymer consisting of repeat units of the type (I). To confirm the polymerization mechanism, NMR spectroscopic analyses of the Diels-Alder reaction and the radical polymerization reaction of furan with MAH were carried out.

Equimolar reaction mixtures of furan derivatives with $\mathrm{MAH}$ in acetone- $d_{6}$ were examined at room temperature by NMR, using nitromethane as an internal standard. A typical example of the NMR spectra of the DielsAlder reaction system is shown in Figure 7, in which the peaks at $5.98 \tau\left(\mathrm{H}_{5}\right.$ and $\left.\mathrm{H}_{6}\right)$ and $4.12 \tau$ $\left(\mathrm{H}_{4}\right)$ due to the exo isomer of the Diels-Alder adduct of MF with $\mathrm{MAH}$, and the peaks at $5.29 \tau\left(\mathrm{H}_{5}\right.$ and $\left.\mathrm{H}_{6}\right)$ and $4.04 \tau\left(\mathrm{H}_{4}\right)$ due to the endo isomer simultaneously appeared with the signal $(4.96 \tau)$ of nitromethane as an internal standard. Accordingly, the percent yields of the exo and the endo isomers can be calculated from the areas under the peaks at 5.98 and $5.29 \tau$ (or 4.12 and $4.04 \tau$ ), respectively.

The percent conversions for the two isomers 


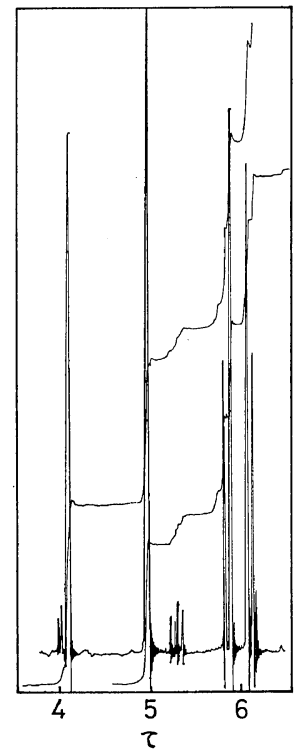

Figure 7. NMR spectrum of an equimolar reaction mixture of MF with $\mathrm{MAH}$ in acetone- $d_{6}$, measured at $23^{\circ} \mathrm{C}$, using nitromethane as an internal standard.

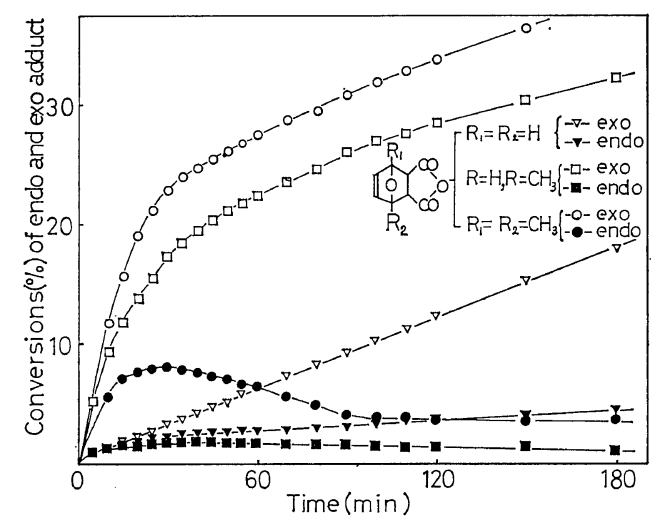

Figure 8. Time-conversion curves of the exo and endo isomers of furan derivatives-MAH in the solution of acetone- $d_{6}$ at $23^{\circ} \mathrm{C}$ : [Furan deriv.], 2.1 $\mathrm{mol} / l$; [MAH], $2.1 \mathrm{~mol} / l$.

of the Diels-Alder adducts of F, MF, and DMF with MAH as a function of time are shown graphically in Figure 8 . The reactions proceed stereospecifically to form preferentially exo isomers, especially in the MF-MAH system. This is the opposite observation to the Alder's "Maxmalle Anhäfung von Doppel Bindungen.", Initially, the exo isomer was formed about twice as fast as the endo isomer, but after about a day, the endo isomer had almost completely disappeared and only exo isomer and small amounts of unreacted furans and MAH remained. The maximum conversions for the endo isomers were observed in every case. This suggests the isomerization of the endo adducts to the exo isomers, which may be thermodynamically more stable.

The initial rates of the formation of the exo adducts increased in the order: $\mathrm{F}<\mathrm{MF}<\mathrm{DMF}$, whereas those of the endo form increased in a different order: $\mathrm{MF}<\mathrm{F}<\mathrm{DMF}$. As shown in Figure 8 , the total conversion for the DMFMAH adduct isomers has already reached approximately $26 \%$ after $20 \mathrm{~min}$. No one ever ignores the participation of the Diels-Alder adduct in the radical polymerization of furan derivatives with MAH.

It is of interest that the order of the polymer yields is consistent with that of the exo isomers formed mainly by the Diels-Alder reaction in the polymerization system. This fact suggests a polymerization through the adduct. Also, as previously shown in Table I, the Diels-Alder adduct itself (exo isomer) was successfully polymerized, and the adduct polymer and the addent polymer contain essentially the identical structural repeating unit of the type (I).

We can thus point out the following additional but undirect supports for the polymerization mechanism through the Diels-Alder adduct.

(1) Furan derivatives could never copolymerize with various vinyl monomers in the presence of radical initiators.

(2) Without decomposition of a radical initiator, furan derivatives could not polymerize but could take part in the Diels-Alder reaction with MAH at a fairly high speed through the formation of the charge-transfer complex species.

To confirm unequivocally the above-mentioned polymerization mechanism, the polymerization reaction was directly followed by NMR spectra.

MAH and catalytic amounts of AIBN were put into a NMR sample tube, which had previously been filled with dry nitrogen.

An equimolar amount of furans in acetone- $d_{6}$ solution were introduced into the tube by using microsyringes. The tube was then connected to a vacuum line, degassed at liquid-nitrogen tem- 
B. Kamo, I. Morita, S. Horie, and S. Furusawa

(a)

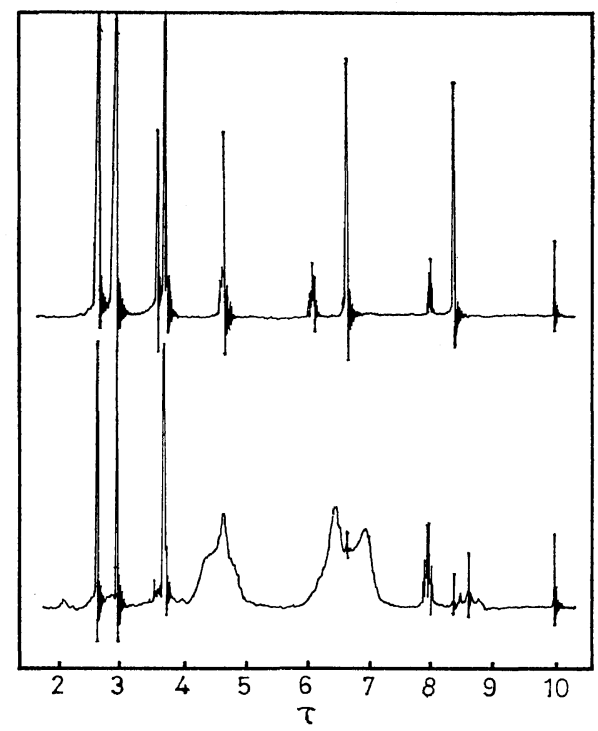

Figure 9. NMR spectra of F-MAH system in the presence of AIBN in acetone- $d_{6}:$ a, after 30 min at room temp; b, after $1 \mathrm{hr}$ at $60^{\circ} \mathrm{C}$. perature, and sealed off. Finally the tube was set in the NMR spectrometer at room temperature. The reaction mixture in a tube was subjected to inspection by NMR spectroscopy at each desired time of reaction. A typical example of the NMR spectra of the reaction between $\mathrm{F}$ and $\mathrm{MAH}$ is given in Figure 9.

Several new peaks at $3.28 \tau\left(\mathrm{H}_{2}\right.$ and $\mathrm{H}_{3}$; multiplet), $4.63 \tau\left(\mathrm{H}_{1}\right.$ and $\mathrm{H}_{4}$; overlapping multiplet) and $6.08 \tau\left(\mathrm{H}_{5}\right.$ and $\mathrm{H}_{6}$; quartet) assigned to the endo adduct, and at $3.37 \tau\left(\mathrm{H}_{2}\right.$ and $\mathrm{H}_{3}$; multiplet), $4.65 \tau\left(\mathrm{H}_{1}\right.$ and $\mathrm{H}_{4}$; multiplet), and $6.68 \tau$ $\left(\mathrm{H}_{5}\right.$ and $\mathrm{H}_{6}$; singlet) due to the exo isomer, simultaneously appeared. The percent conversion of $\mathrm{F}$ can be calculated from the peak area of methine protons of unreacted $F(3.47 \tau)$. After allowing to stand the reaction was followed by NMR spectra at an elevated temperature of $60^{\circ} \mathrm{C}$. The signal $(8.2 \tau)$ of the methyl protons of the AIBN catalyst was found to disappear completely

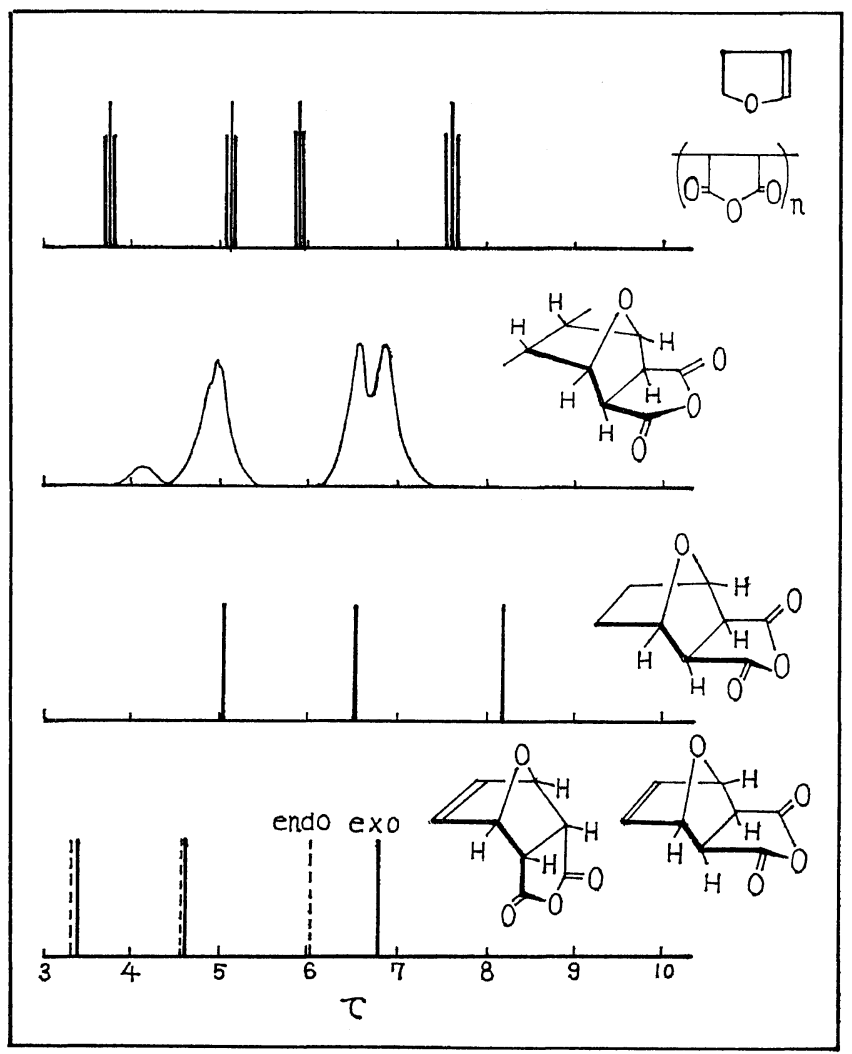

Figure 10. Comparisons of the NMR spectra of the polymer obtained by the radical polymerization of $\mathrm{F}$ and $\mathrm{MAH}$ with that of its model compounds. 


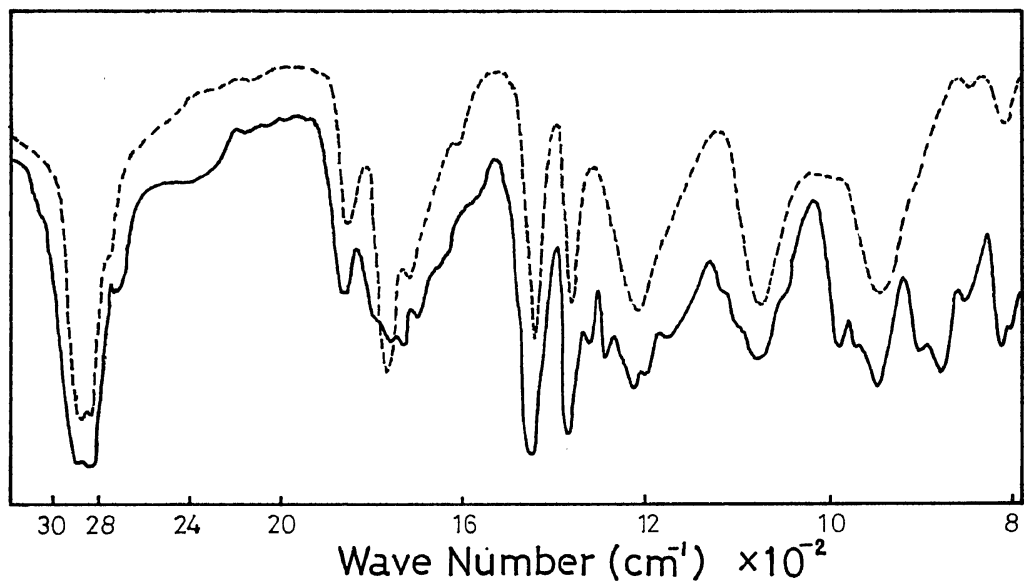

Figure 11. IR spectra of poly(exo F-MAH adduct) and exo HPA: Full line, poly(exo F-MAH adduct); dotted line, exo HPA.

and new broad absorptions appeared at $4.59,6.5,1$ and $6.80 \tau$.

The chemical shifts of these three peaks were quite identical with those of the addent and the adduct polymer resulting from $\mathrm{F}$ and $\mathrm{MAH}$, as shown in Figure 2 and Table II. In addition to these absorptions, the peaks due to unreacted monomers and the Diels-Alder adducts also still remained.

The comparisons of the NMR spectra of the $\mathrm{F}-\mathrm{MAH}$ system in Figure 9 with those of the model compounds are schematically shown in Figure 10.

If the chemical structure of the polymerization product could be (II), Butler, et al., suggested, at least five kinds of absorption peaks should be observed. Four peaks should appear at $4-5 \tau$, since the peaks of the model compound, 2,3-dihydrofuran, appeared in similar regions (3.76 and $5.14 \tau$, olefinic; 5.85 and $7.53 \tau$, methylene $)^{8}$ and a peak due to MAH repeating units should also be observed at 5.8 $\tau$. But no absorption peaks corresponding to the above were observed. Hence, the reaction product must have a structure other than (II). These results and the area intensities of the peaks support the assertion that the structural units of the polymer are (I).

The IR spectrum of the polymer was also found to contradict the structure (II), as indicated in Figure 11. Strong, broad bands of the ether linkage in 2,3-dihydrofuran could not be found in the polymer spectrum. Moreover, dihydrofuran units can not be expected to remain in stable form in the polymer, since it is so reactive as to give a homopolymer in the presence of a trace of acid and also to give an alternative copolymer in the presence of MAH without any initiators. ${ }^{8}$ Dihydrofuran units in the copolymer chain, if any, might react with unconverted MAH in the polymerization system. From the similarity of the spectra between the polymer and the exo isomer of the Diels-Alder adduct resulting from $\mathrm{F}$ and $\mathrm{MAH}$, we should like to support $(\mathbf{I})$ as the chemical structure of the polymer.

From all of the experimental results mentioned above, we conclude that furan derivatives and MAH do not ungergo free radical copolymerization to yield a $1: 1$ alternating copolymer, but ordinarily take part in the Diels-Alder reaction, and the resulting adducts are homopolymerized by radical initiators.

\section{Copolymerization of the Exo Adduct}

Radical copolymerization of the exo isomer of the Diels-Alder adduct between F and MAH was carried out to find out experimentally whether the double bond of the adduct has polymerizability or not. The values of monomer reactivity ratio, $r_{1}$ (exo adduct) $=0.24, r_{2}$ (styrene $)=2.38$, were determined by using the Mayo-Lewis scheme.

The exo adduct was found to revert partly to its component in the polymerization system. 


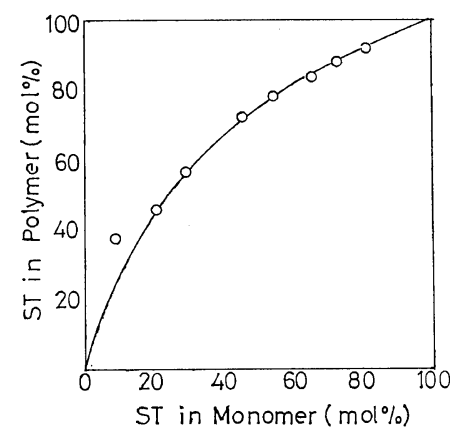

Figure 12. Copolymer composition curve of exo $\mathrm{F}-\mathrm{MAH}$ adduct $\left(\mathrm{M}_{1}\right)$ and styrene $\left(\mathrm{M}_{2}\right)$ : Catalyst, AIBN; temp, $70^{\circ} \mathrm{C}$; solvent, acetone.

The concentration of the adduct, however, was still high enough in the early stage of the reaction. It seems to be obvious from the IR and NMR spectra and the composition analysis of the resulting copolymer that in the copolymerization reaction of the exo adduct with styrene, copolymerization of styrene with MAH formed by reversion of the adduct rarely takes place. The above results suggest that the exo adduct with an internal double bond may add to its own radical as well as styryl radical.

\section{Reaction of Furfuryl Methacrylate (FMA) with $M A H$}

Let us take another such example in which the Diels-Alder adduct takes part in the polymerization reaction. A calculated quantity of FMA is added to MAH suspended in ether. After the reaction mixture is refluxed for several hours, it is allowed to stand for a night in the cold in order to complete the reaction, during which time the addition product separated for the most part in the form of hard, colorless crystals. The product was found to be an adduct of FMA and MAH, which was previously unknown.

From the IR and NMR spectra analyses (Figure 13), this new adduct was found to be a highly stereospecific structure of exo form.

In the presence of $A I B N$, the adduct reacts at $60^{\circ} \mathrm{C}$ in benzene solution to give a white powdered polymer. The chemical structure of the polymer was the vinyl polymerization product and the pendant furan nuclei did not take part in the reaction.

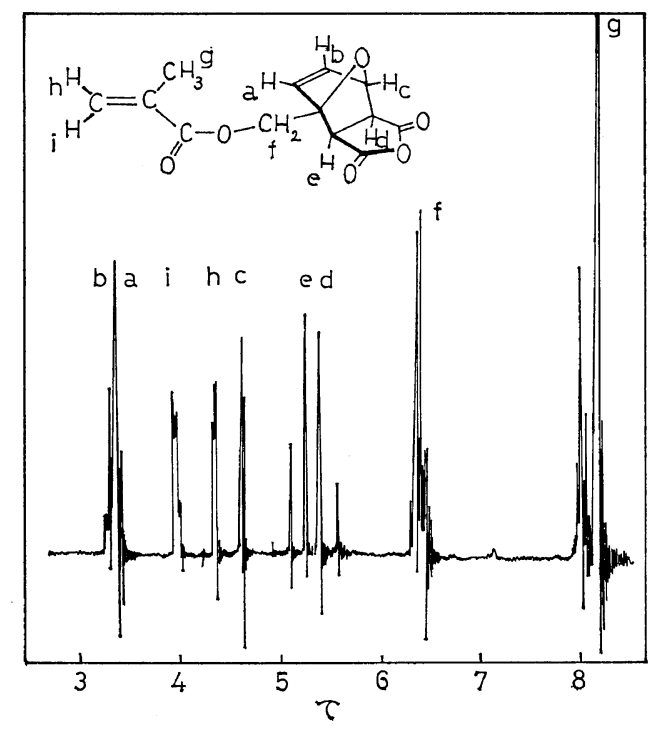

Figure 13. NMR spectrum of exo isomers of the Diels-Alder adduct resulting from FMA with MAH, measured in acetone- $d_{6}$ solution $(20 \mathrm{wt} / \mathrm{vol} \%)$ at $23^{\circ} \mathrm{C}$.

The IR and NMR spectra, and the elementary analysis suggest the polymer structure to be:

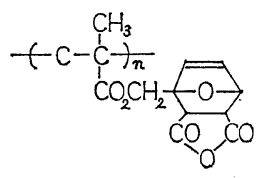

The identical polymer was also obtained when equimolar amounts of FMA and MAH were polymerized in the presence of AIBN in a benzene solution. These results suggest that a newly formed internal double bond of furan nuclei in the FMA-MAH adduct does not participate in the polymerization reaction because of its lower reactivity to a radical than that of the vinyl group of the methacrylate. Details will be discussed elsewhere.

\section{REFERENCES}

1. B. Kamo, K. Ito, and S. Furusawa, Preprints of 21th Annual Meeting of the Chem. Soc. of Japan, Osaka, May 1968, No. 16208.

2. G. B. Butler, J. T. Badgett, and M. Sharabash, J. Macromol. Sci. Chem., A4(1), 51 (1970).

3. O. Diels and K. Alder, Ber., 62, 554 (1929). 
4. J. S. Fritz and N. M. Lisicki, Anal. Chem., 23, 589 (1951).

5. P. Job, Ann., 9, 113 (1928).

6. S. Iwatsuki and Y. Yamashita, Makromol. Chem., 89, 205 (1965).

7. W. G. Barb, Trans. Faraday Soc., 49, 143 (1953).

8. B. Kamo, S. Kurashige, and S. Furusawa,
Kogyo Kagaku Zasshi (J. Chem. Soc. Japan, Ind. Chem. Sect.), 73, 580 (1970).

9. F. A. L. Anet, Tetrahedron Lett., 1219 (1962).

10. H. Staudinger, Ann., 467, 73 (1928).

11. K. Ito, B. Kamo, and S. Furusawa, Preprints of 20th Annual Meeting of the Chem. Soc. of Japan, Tokyo, March 1967, No. 40018. 